Guy Lepage

C.M. Frank Kneepkens Claude C. Roy
ADRESSES ET TIRÉS A PART

G. Lepage : étudiant au doctorat, C.-C. Roy Unité de recherche en gastroentérologie-nutrition, Directeur du Département de pédiatrie, Hôpital Sainte-Justine, 3175 Chemin de la Côte SteCatherine, Montréal (Québec) H3T 1C5 CM Frank Kneepkens : professeur-adjoint, département de pédiatrie, Free University Hospital, Amsterdam, The Netherlands.

\section{Dysfonction peroxysomiale associée à la déficience en acides gras essentiels}

Les peroxysomes sont des organites intracellulaires responsables de nombreuses fonctions métaboliques essentielles. Une dysfonction peroxysomiale peut survenir à la suite d'anomalies dans la biogenèse et dans l'assemblage de ces organites. Cependant, elle peut aussi être la conséquence de certains dérèglements métaboliques. Nous avons constaté que près de $50 \%$ des enfants atteints de mucoviscidose présentent des signes biochimiques de déficience en acides gras essentiels et nous avons noté que les phospholipides érythrocytaires subissaient à la fois une augmentation de leurs acides gras à chaîne longue et une diminution de leurs plasmalogènes : ces deux constatations suggèrent un dérèglement de la fonction peroxysomiale. Les résultats de ces expériences ont conduit au développement d'un modèle animal de déficience en acides gras essentiels qui nous a permis de reproduire les signes précurseurs de dysfonction peroxysomiale. Enfin, un supplément en $\beta$-carotène introduit dans le régime alimentaire de 12 enfants atteints de mucoviscidose a eu pour effet non seulement d'éliminer une lipoperoxydation consécutive au déséquilibre entre pro- et anti-oxydants mais encore de restaurer leur fonction peroxysomiale, tout en améliorant leurs niveaux d'acides gras essentiels. Ces résultats permettent d'émettre l'hypothèse suivant laquelle la peroxydation lipidique pourrait en partie être responsable de la déficience en acides gras essentiels ; de plus, cette déficience pourrait entraîner une dysfonction peroxysomiale. La compréhension de ces mécanismes aura sans doute d'importantes répercussions sur le plan clinique et ouvrira la voie à de nouvelles perspectives de traitement de cette maladie. 


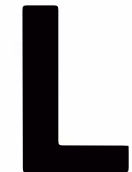

es peroxysomes sont des organites intracellulaires que l'on trouve en grandes quantités dans le monde végétal et dans le monde animal [1]. Ils jouent un rôle dans de nombreuses fonctions cellulaires. $\mathrm{Au}$ cours des dernières années, la $\beta$ oxydation des acides gras, la biosynthèse des plasmalogènes, la formation des acides biliaires et l'activité antioxydante ont tout particulièrement fait l'objet d'études [2].

De Duve découvrit les peroxysomes en 1965 [3]. Par la suite, aucun progrès réel concernant la compréhension de leur rôle ne fut réalisé avant que Goldfischer et al. constatent que les peroxysomes ne se trouvent ni dans le foie ni dans le rein d'enfants souffrant du syndrome cérébrohépatorénal de Zellweger [4]. Depuis ce temps, beaucoup d'autres maladies affectant l'intégrité des peroxysomes ont permis d'identifier des anomalies dans la biogénèse et dans l'assemblage de ces organites, ainsi que des déficiences enzymatiques particulières modifiant leur mode d'action [5]. Une dysfonction peroxysomiale accompagne parfois les pathologies mitochondriales [6] ; elle peut également survenir à la suite de certaines déficiences métaboliques comme la malnutrition [7].

Au cours d'une récente étude, nous avons rassemblé une série de faits qui montrent qu'un certain nombre d'enfants atteints de mucoviscidose présentent des signes biochimiques de dysfonction peroxysomiale. Nous présentons ici un bref survol des principales recherches en cours dans le but de situer le problème dans le contexte général des anomalies métaboliques observées dans la mucoviscicose et de discuter des hypothèses de travail qui servent de base à nos propres recherches.

\section{Mucoviscidose et déficience en acides gras essentiels}

La déficience en acides gras essentiels est une complication de la mucoviscidose, que l'on connaît depuis 30 ans [8]. On l'a attribué à une insuffisance pancréatique, un régime alimentaire pauvre en lipides ainsi qu'à une ingestion calorique insuffi- sante par rapport à l'augmentation des besoins. Les tentatives pour corriger cette situation ont largement échoué [9]. Un groupe de chercheurs a attribué la déficience en acides gras essentiels à une anomalie du métabolisme de l'acide arachidonique [10] mais cette anomalie n'a pas été confirmée (Maarten Sinaasappel, communication personnelle). Il se pourrait, bien sûr, qu'il existe un rapport de cause à effet avec la protéine CFTR anormale.

Si les prochains travaux sur la mucoviscidose montraient qu'il existe un turnover accéléré des acides gras essentiels et particulièrement de l'acide arachidonique, il se pourrait die soient le résultat à la fois d'une surproduction d'eicosanoïdes et de la déficience en acides gras essentiels.

Au cours d'une étude de dépistage portant sur 163 enfants atteints de mucoviscidose, on a noté que près de que certains symptômes de la mala-

$50 \%$ d'entre eux présentaient des signes biochimiques de déficience en acides gras essentiels, si l'on se réfère au rapport (C20 : 3n9/C20 : 4n6) décrit par Holman [11]. Cet index tient compte à la fois de la concentration de l'acide eicosatriénoique (C20 : 3n9), métabolite de l'acide oléique qui augmente invariablement et de celle de l'acide arachidonique (C20 : 4n6), métabolite le plus important de l'acide linoléique.

\section{Répercussions possibles de la déficience en acides gras essentiels}

On sait que les acides gras essentiels entrent dans la composition des membranes, déterminent la production des eicosanoïdes et influencent la qualité de la réponse immunitaire de la cellule [12]. La déficience biochimique observée plus haut s'accompagne de modifications des phospholipides membranaires des globules rou-

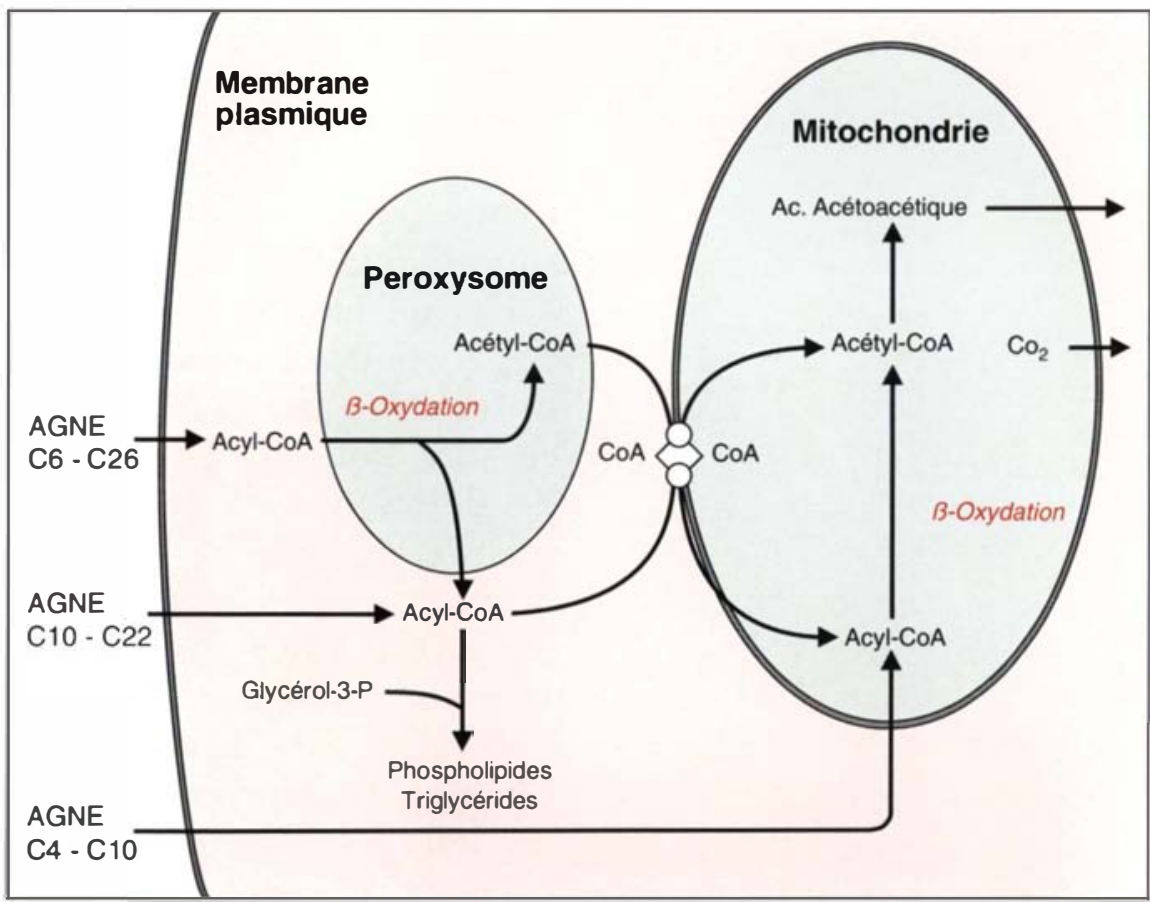

Figure 1. Schéma de la $\beta$-oxydation des acides gras. Le métabolisme des acides gras dans le foie dépend de la longueur de la chaîne de carbone. Alors qu'un acide gras de 10 carbones ou moins peut entrer directement dans la mitochondrie, il devra être activé en acyl-CoA dans le réticulum endoplasmique ou dans la membrane mitochondriale externe si la chaîne dépasse 10 carbones. Le triangle sur la membrane mitochondriale représente la dépendance des acides gras envers la carnitine pour leur transfert à l'intérieur de la mitochondrie. Par contre, si l'acide gras comporte plus de 22 carbones, il

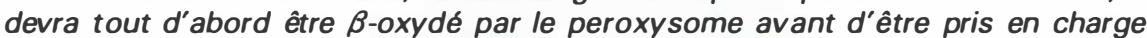
par la mitochondrie. AGNE, acide gras non estérifié; CoA, coenzyme A; glycérol-3-P, glycérol-3-phosphate; ac. acétoacétique, acide acétoacétique. 
ges et des plaquettes [13] et altère leur mode d'action [14]. Pourrait-on attribuer à cette déficience le transport tubulaire anormal du $\mathrm{Na}+$ [15] ou la dysfonction mitochondriale décrite dans la mucoviscidose [16] ? Telles sont les questions auxquelles il nous faut répondre.

Les conséquences cliniques de ces observations sont considérables; en effet, nos travaux expérimentaux ont montré que la déficience en acides gras essentiels cause chez le rat Sprague-Dawley une diminution de l'absorption des lipides en affectant à la fois la phase digestive (diminution de la sécrétion des acides biliaires) et la phase absorptive, mesurée in vitro dans des explants en culture [17].

A la suite de ces observations, il faut donc se demander si la diminution du taux de sécrétion abaissé des acides biliaires dans la mucoviscidose [18] s'expliquerait non seulement par les pertes excessives d'acides biliaires dans les selles [19] mais encore par une réduction de leur synthèse. $\mathrm{Au}$ problème de la phase digestive, s'ajouterait donc un déficit du transport et de la synthèse des lipoprotéines intestinales relié à la déficience en acides gras essentiels qui, comme on l'a vu plus haut, affecte près de $50 \%$ des malades.

\section{La déficience en acides gras essentiels entraîne une dysfonction peroxysomiale}

L'année dernière (1992) les données que nous avons recueillies auprès d'enfants souffrant de mucoviscidose nous ont permis de montrer qu'un certain nombre d'entre eux présentent un profil lipidique plasmatique caractérisé par une augmentation nette d'acides gras à chaîne très longue ( $>$ C22) dont la $\beta$-oxydation est sous la dépendance du peroxysome (figure 1) (soumis pour publication, G.L., N. Ronco, J. Champagne, C.C.R.). Intrigués par cette observation, nous avons alors étudié les phospholipides des globules rouges où nous avons constaté une diminution des plasmalogènes. On sait que les 2 premières réactions enzymatiques responsables de la synthèse de cette famille de phospholipides qui diffèrent

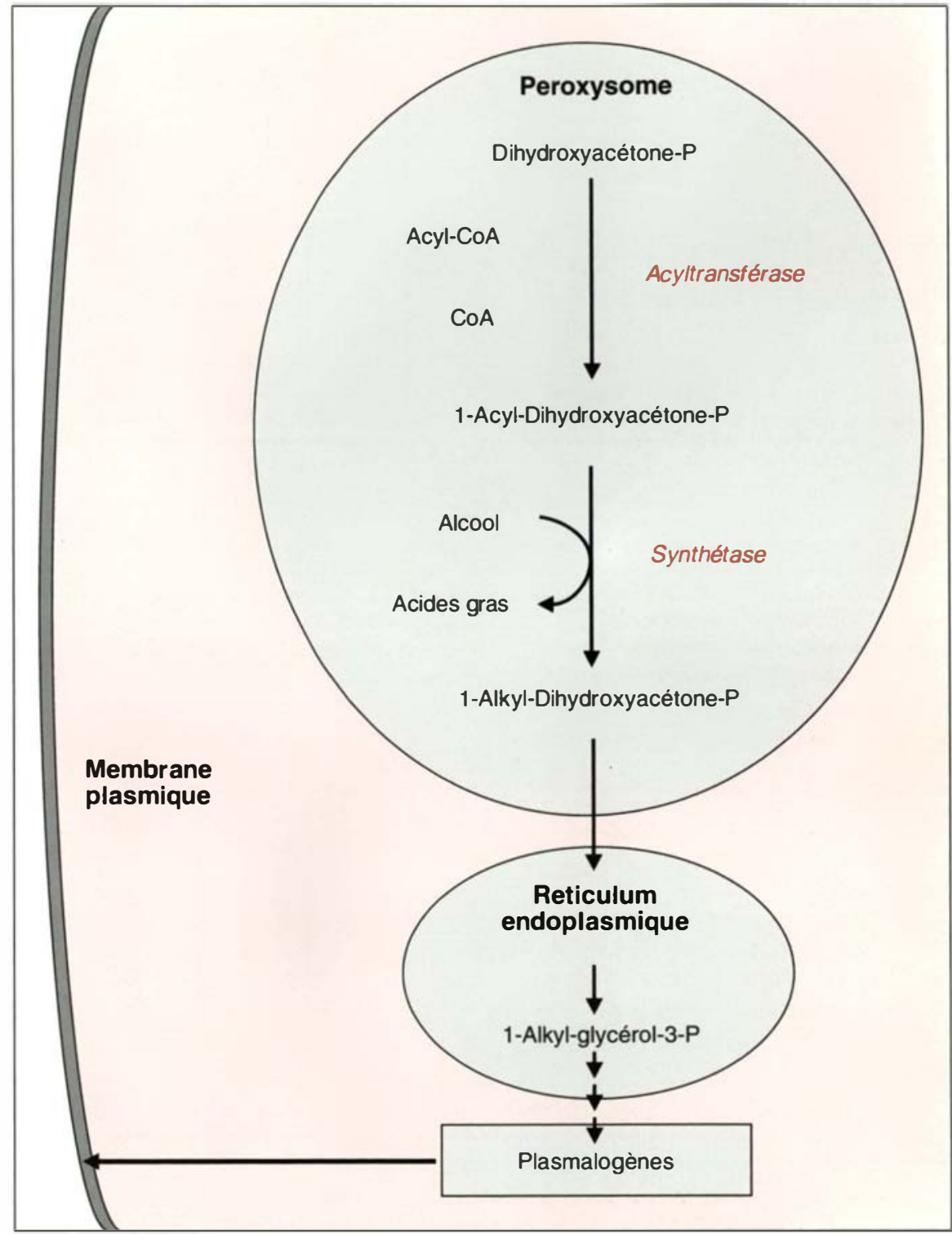

Figure 2. Localisation intracellulaire des enzymes responsables de la synthèse des plasmalogènes. L'acyl-CoA : dihydroxyacétone-phosphate acyltransférase et le 1-alkyl-dihydroxyacétone-phosphate synthétase sont les deux premiers enzymes responsables de la synthèse des plasmalogènes. Ils sont situés dans la membrane interne du peroxysome. CoA, coenzyme A.

des autres par la présence d'une liaison éther au lieu d'un lien ester, requièrent une fonction peroxysomiale normale (figure 2).

Nous avons remarqué que seuls les patients affectés d'une déficience en acides gras essentiels présentaient ces anomalies biochimiques. Cette observation ouvrait la perspective d'une altération des fonctions métaboliques des peroxysomes. Pour mettre en évidence cette altération, nous avons créé un modèle animal de déficience en acides gras essentiels (soumis pour publication, G.L., N. Ronco,
J. Champagne, C.C.R.). Nous avons nourri des rats Spraque-Dawley de telle sorte que le degré de déficience corresponde à celui observé dans la mucoviscidose. La figure 3 qui regroupe les résultats de l'étude clinique (figure $3 A$ ) et de l'étude expérimentale (figure $3 B$ ) permet de supposer que la dysfonction peroxysomiale observée dans la mucoviscidose est causée par la déficience en acides gras essentiels et n'a probablement pas de rapport avec le CFTR mutant même si l'absence d'acidification des organites qui a été rapporté [20] 

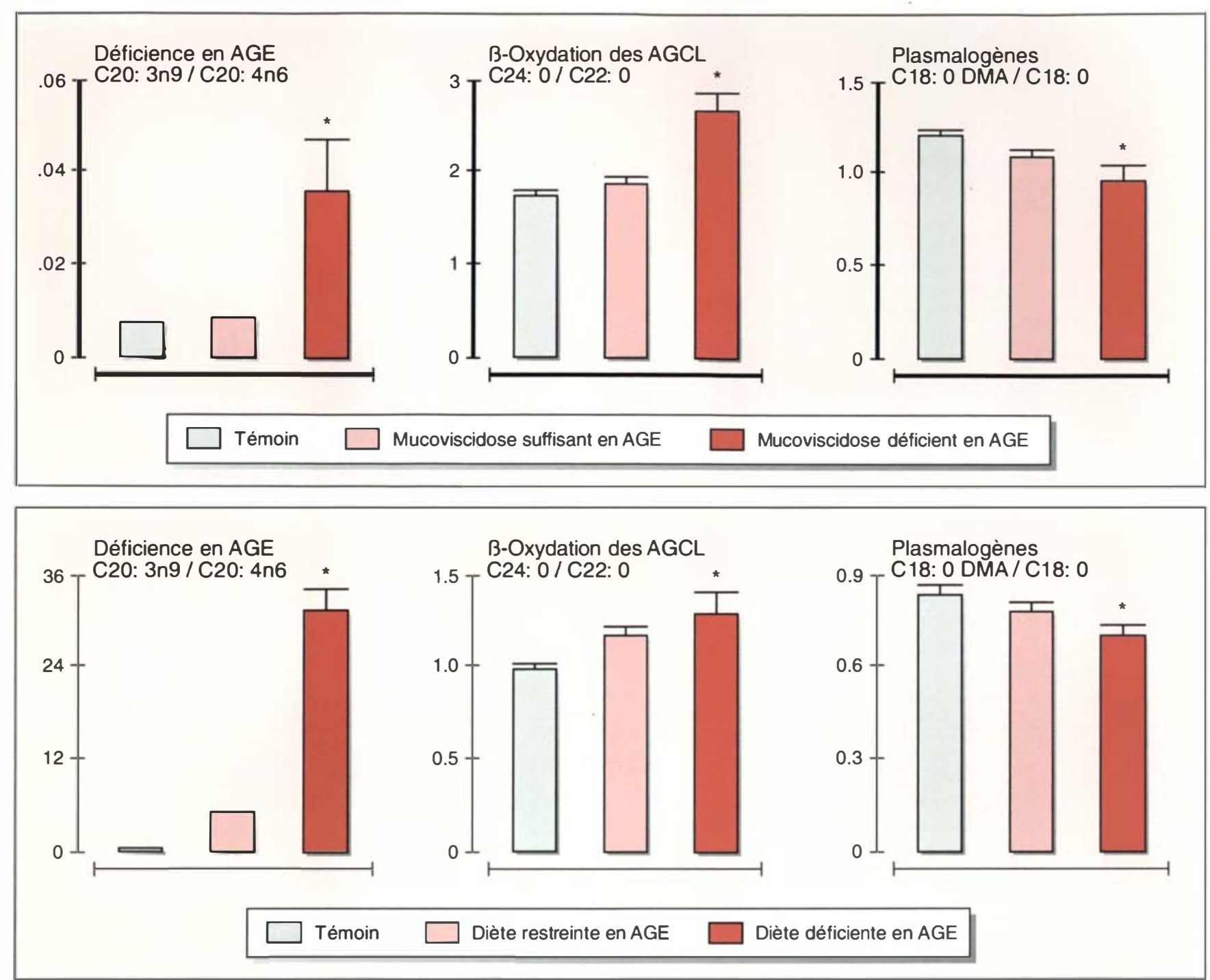

Figure 3. Effets de la déficience en acides gras essentiels sur la fonction peroxysomiale chez les patients atteints de mucoviscidose (3A) et dans le modèle animal (3B). AGE, acides gras essentiels; AGCL, acides gras à chaîne longue; DMA, diméthylacétal. Le groupe déficient en AGE est statistiquement différent (P< 0.05$)$ des deux autres groupes par étude ANOVA.

expliquerait peut-être nos observations.

\section{Dysfonction peroxysomiale} et métabolisme anormal des acides biliaires

Dans les maladies peroxysomiales, le métabolisme des acides biliaires est profondément altéré ; en effet, le peroxysome joue un rôle clé dans l'oxydation de la chaîne latérale des deux précurseurs de l'acide chénodéoxycholique et cholique [21] : l'acide déhydroxycoprostanique et trihydroxycoprostanique respectivement (figure 4). Or, nous avons constitués de 27 atomes de carbone à la place des acides biliaires primaires qui comptent 24 atomes de carbone. Une étude récente chez le rat permet de supposer que le peroxysome joue un rôle important non seulement dans la synthèse des acides biliaires primaires mais encore dans leur amidation en particulier avec la taurine [22]. Or nos résultats, confirmés par plusieurs équipes de recherche, montrent une forte prédominance d'acides biliaires amidés à la glycine chez les patients atteints de mucoviscidose [23]. Le ratio très élevé d'acides biliaires glycoconjugués/tauroconjugués attribué à des pertes excessives de taurine dans les selles, consti- tuerait donc une manifestation supplémentaire de la dysfonction peroxysomiale.

\section{Mucoviscidose et peroxydation lipidique}

Les effets pathologiques des radicaux libres font l'objet de nombreuses recherches. Le nombre de ces radicaux grandit en l'absence d'une augmentation compensatrice des antioxydants endogènes (enzymes et autres molécules) et exogènes ( $\alpha$-tocophérol, $\beta$-carotène, etc.). Leurs effets nocifs se traduisent sous la forme de dommage cellulaire, d'altération des membranes et de leurs protéines, 
ạinsi que de dégradation de l'ADN. Étant donné l'inflammation pulmonaire chronique, la malabsorption des vitamines liposolubles et la malnutrition fréquente, il n'est pas surprenant qu'on ait observé des signes d'attaque radicalaire [24]. Nous avons confirmé ces données en mesurant deux marqueurs de peroxydation lipidique, le malondialdéhyde et l'exhalaison d'éthane et de pentane dont nous avons raffiné la spécificité, la précision et la reproductibilité $[25,26]$. Comme dans le cas de la dysfonction

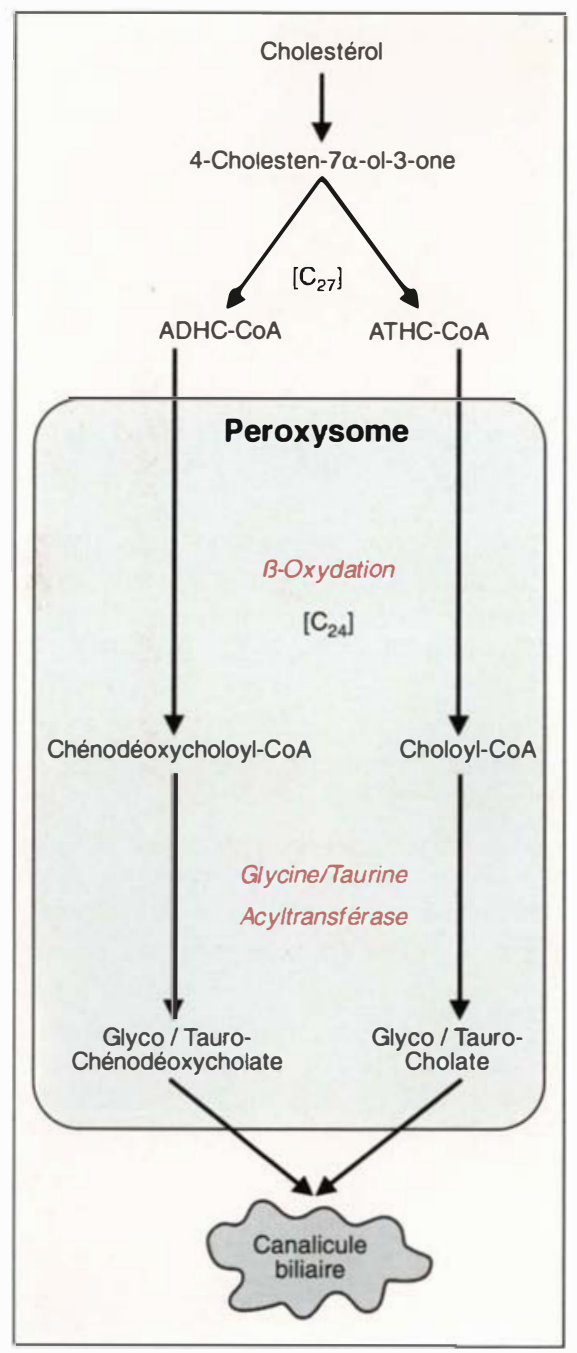

Figure 4. Schéma simplifié du mécanisme de formation des acides biliaires. Les états précurseurs $d^{\prime}$ acides biliaires doivent d'abord être $\beta$-oxydés dans le peroxysome avant d'être conjuguées à la glycine où à la taurine. Par la suite, les acides biliaires conjugués seront exportés vers le canalicule biliaire. ADHC, acide dihydrocoprostanique; $A T H C$, acide trihydrocoprostanique; CoA, coenzyme $A$. peroxysomiale, la peroxydation lipidique semblait associée à la quantité insuffisante en acides gras essentiels.

\section{La dysfonction peroxysomiale pourrait-elle contribuer à la peroxydation lipidique?}

Étant donné que les radicaux libres attaquent les doubles liaisons des acides gras et de façon préférentielle ceux qui sont polyinsaturés, il est étonnant de noter que la peroxydation lipidique était augmentée chez les patients qui subissaient une baisse en acides gras polyinsaturés.

On pourrait résoudre ce paradoxe en supposant que la peroxydation lipidique constitue une fois de plus une manifestation de la dysfonction peroxysomiale. Des travaux récents ont prouvé, en effet, que l'activité catalasique et la dismutase dépendante du cuivre et du zinc se retrouvent exclusivement dans le peroxysome [27]. Une diminution du nombre des peroxysomes et de leur capacité antioxydante pourrait donc entraîner une peroxydation lipidique. Une étude clinique dont les résultats n'ont pas encore été confirmés montre qu'en ajoutant du $\beta$ carotène ( $15 \mathrm{mg}$ t.i.d.) au traitement de 12 patients atteints de mucoviscidose non seulement on corrige la lipoperoxydation mais encore on améliore la fonction peroxysomiale [28]. Ces résultats ne nous permettent pas de conclure, car chez ces malades, le $\beta$-carotène pourrait s'avérer être un anti-oxydant exogène nécessaire, même si les taux d'alphatocophérol sont normaux. Par ailleurs, le fait que le rat déficient en acides gras essentiels et ayant reçu une dose supplémentaire de $\alpha$ tocophérol et de $\beta$-carotène présente malgré tout des signes biochimiques de dysfonction peroxysomiale vient appuyer notre hypothèse.

\section{La peroxydation lipidique pourrait-elle contribuer à la déficience en acides gras essentiels ?}

On sait que les antioxydants comme l'alpha-tocophérol inhibent la phospholipase $\mathrm{A}_{2}[29]$ et modulent l'acti-

\section{RÉFÉRENCES}

1. Lazarow PB. The Liver : Biology and Pathobiology. New York: Raven Press, Ltd., 1988 ; 241-54.

2. Vamecq J, Poll-The BT, Saudubray JM. Biochimie du peroxysome et maladies peroxyzomiales. Ann Biol clin 1988; 46 : 233-58.

3. De Duve C, Baudhuin P. Peroxisomes (microbodies and related particles). Physiol Rev 1966 ; 46 : 323-57.

4. Goldfischer S, Moore CL, Johnson AB et al. Peroxisomal and mitochondrial defects in the cerebro-hepato-renal syndrome. Science $1972 ; 182: 62-4$.

5. Moser HW. Peroxisomal disorders. Clin Biochem 1991; 24 : 343-51.

6. Goldfischer SL. The Liver: Biology and Pathobiology. New York: Raven Press, Ltd., 1988 ; 255-67.

7. Doherty JF, Golden MHN, Brooks SEH. Peroxisomes and the fatty liver of malnutrition : an hypothesis. Am J Clin Nutr $1991 ; 54: 674-7$.

8. Kuo PT, Huang NN, Bassett DR. The fatty acid composition of the serum chylomicrons and adipose tissue of children with cystic fibrosis of the pancreas. J Pediatr $1962 ; 60$ : 394-403.

9. Dodge JA, Custance JM, Goodchild MC, Laing SC, Vaughan M. Paradoxical effects of essential fatty acid supplementation on lipid profiles and sweat electrolytes in cystic fibrosis. $B r J$ Nutr $1990 ; 63$ : 259-71.

10. Strandvik B. Relation between essential fatty acid metabolism and gastrointestinal symptoms in cystic fibrosis. Acta Paediatr Scand Suppl $1989 ; 363$ : 58-65.

11. Holman RT. The ratio of trienoic: tetraenoic acids in tissue lipids as a measure of essential fatty acid requirement. $J$ Nutr $1960 ; 70$ : 405-410

12. Dvorak B, Stepankova R. Effects of dietary essential fatty acid deficiency on the development of the rat thymus and immune system. Prostaglandins Leukot Essent Fatty Acids 1992 ; 46 : 183-90.

13. Lepage G, Levy E, Ronco N, Smith L, Galeano N, Roy CC. Direct transesterification of plasma fatty acids for the diagnosis of essential fatty acid deficiency in cystic fibrosis. J Lipid Res 1989 ; 30 : 1483-90.

14. Sigström L, Strandvik B. Erythrocyte sodium-potassium transport in cystic fibrosis. Pediatr Res 1992 ; 31 : 425-7.

15. Strandvik B, Bronnegard M, Gilljam H, Carlstedt-Duke J. Relation between defective regulation of arachidonic acid release and symptoms in cystic fibrosis. $J$ Gastroenterol 1988 ; 23 (Suppl. 143) : 1-4.

16. Shapiro BL. Evidence for a mitochondrial lesion in cystic fibrosis. Life Sciences $1989 ; 44: 1327-34$

17. Levy E, Garofalo C, Thibault L et al. Intraluminal and intracellular phases of fat absorption are impaired in essential fatty acid deficiency. Am J Physiol 1992; 262 : G319-G26. 
vité de la cyclo-oxygenase [30]. Le $\beta$ carotène, comme tel, a été moins bien étudié ; cependant, il a sûrement joué un rôle important dans notre étude : il corrige la lipoperoxydation et il améliore la dysfonction peroxysomiale. De plus, on a noté qu'il a augmenté les niveaux de vitamine $\mathrm{E}$ et amélioré le profil des acides gras essentiels chez la plupart de nos

malades. La boucle semble donc fermée (figure 5).

\section{Conclusions}

L'importance des peroxysomes en tant qu'organites distincts possèdant des fonctions biochimiques et physiologiques uniques est incontestable. Nos travaux récents soulignent le fait

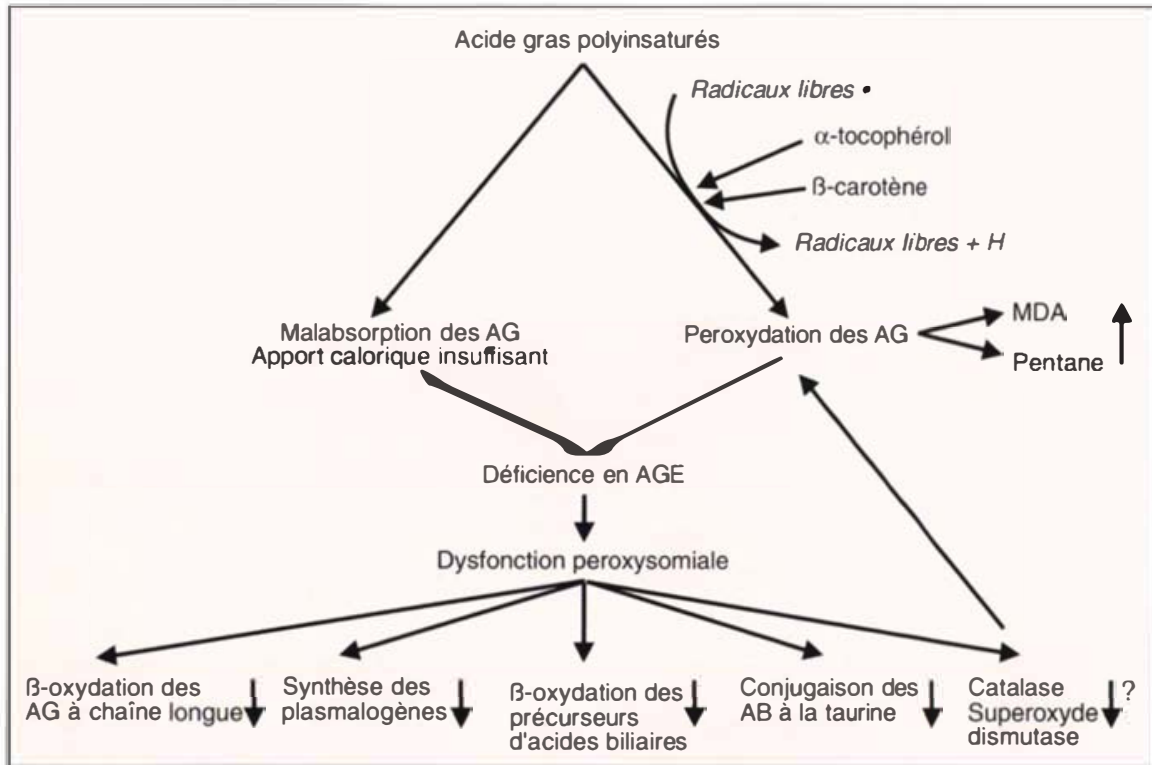

Figure 5. Schématisation des interrelations entre les acides gras polyinsaturés, la déficience en acides gras essentiels (AGE) et la dysfonction peroxysomiale. A noter que l'hypothèse voulant que la peroxydation des lipides secondaire à une attaque radicalaire contribue à la déficience en AGE n'est pas encore prouvée. $A G$, acides gras; MDA, malondialdéhyde; $A G E$, acides gras essentiels; $A B$, acides biliaires.

\section{RÉFÉRENCES}

18. Weber AM, Roy CC. Bile acid metabolism in children with cystic fibrosis. Acto Paediatr Scand 1985 ; Suppl. 317 : 9-15.

19. Weber AM, Roy CC, Morin CL, Lasalle R. Malabsorption of bile acids in children with cystic fibrosis. $N$ Engl J Med 1973 ; 289 : 1001-5.

20. Barasch J, Kiss B, Prince A, Saiman L, Gruenert D, Al-Awqati $Q$. Defective acidification of intracellular organelles in cystic fibrosis. Nature 1991; 352: 70-73.

21. Björkhem I. Mechanism of degradation of the steroid side chain in the formation of bile acids. J Lipid Res 1992 ; 33 : 455-71.

22. Kase BF, Björkhem I. Peroxisomal bile acid-CoA : amino-acid $\mathrm{N}$-acyltransferase in rat liver. J Biol Chem 1989 ; 264 : 9220-3. 23. Roy CC, Weber AM, Morin CL et al. Abnormal biliary lipid composition in cystic fibrosis : Effect of pancreatic enzymes. $N$ Engl J Med 1977 ; 297 : 1301-5.

24. Berger $M$. Inflammation in the lung in $\mathrm{CF}$. A vicious cycle that does more harm than good? In : Moss RB, ed. Cystic fibrosis. Infection, Immunopathology and Host Res ponse. Clin Rev Allergy 1991; 9 : 119-42.
25. Lepage G, Munoz G, Champagne J, Roy CC. Preparative steps necessary for the accurate measurement of malondialdehyde by high-performance liquid chromatography. Anal Biochem 1991; 197: 277-283.

26. Kneepkens CMF, Ferreira C, Lepage G, Roy CC. The hydrocarbon breath test in the study of lipid peroxidation : principles and practice. Clin Invest Med $1992 ; 15$ $163-86$.

27. Keller GA, Warner TG, Steimer KS, Hallewell $\mathrm{RA} . \mathrm{Cu}, \mathrm{Zn}$ superoxide dismutase is peroxisomal enzyme in human fibroblast and hepatoma cells. Proc Natl Acad Sci USA $1991 ; 88: 7381-5$

28. Lepage G, Ronco N, Champagne J, Lamarre A, Roy CC. Beta-carotene inhibits lipid peroxidation and improves peroxisomial function in cystic fibrosis (CF). FASEB J $1993 ; 7$ : A

29. Behrens WA. Dietary vitamin E inhibit mitochondrial rat liver phospholipase A. FASEB J 1991 ; 5 : A943.

30. Reddanna P, Whelan J, Burgess JR et al. The role of vitamin $E$ and selenium on arachidonic acid oxidation by way of the 5-lipoxygenase pathway. Ann N Y Acad Sci $1989 ; 570: 136-45$ qu'une déficience biochimique en acides gras essentiels pourrait en modifier le fonctionnement. La recherche en cours tente d'accréditer cette hypothèse par une approche plus réductionniste dont le but est de montrer que l'intégrité morphologique et fonctionnelle du peroxysome est essentielle pour qu'il puisse aider l'organisme à se défendre contre l'attaque des radicaux libres

\section{Remerciements}

Nous remercions Nancy Ronco, Josée Champagne et Geneviève Ménard.

\section{Summary}

Peroxisomal dysfunction associated with essential fatty acid deficiency

Peroxisomes are intracellular organelles responsible for several essential metabolic functions. Peroxisomal dysfunction may be secondary to defects in the biogenesis and/or assembly of peroxisomes, but it may also be secondary to metabolic disorders. Having shown that nearly half of our pediatric Cystic Fibrosis (CF) population had biochemical evidence of essential fatty acid (EFA) deficiency, we then noted from the phospholipid analysis of red cells an increase in very long chain fatty acids as well as a decrease in plasmalogens, two markers suggestive of a disorder in the integrity of peroxisomes. These results then led us to create an EFA deficient animal model which enabled us to reproduce these indices of peroxisomal dysfunction. Finally, $\beta$-carotene supplementation (15 mg t.i.d.) in twelve CF patients, not only corrected lipid peroxidation, secondary to an imbalance between pro- and antioxydants but also peroxisomal function while improving the EFA status. These data raise the hypothesis that lipid peroxidation may be in part responsible for EFA deficiency and may lead to peroxisomal dysfunction. The understanding of these mechanisms has important clinical implications since it may lead to new therapeutic strategies. 